\title{
Acute Exotropia Revealing Lyme Neuroborreliosis in an 8 Years-Old Child
}

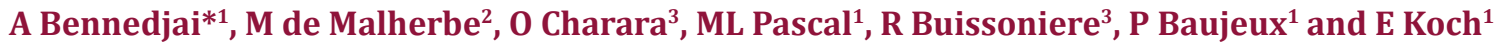 \\ ${ }^{1}$ Department of Ophthalmology, France \\ ${ }^{2}$ Department of Radiology, France \\ ${ }^{3}$ Department of Pediatrics, France
}

Received: 阱: December 12, 2018; Published: 制: January 09, 2019

*Corresponding author: Amin Bennedjai, Department of Ophthalmology, Ch Versailles, France

\begin{abstract}
Introduction: Neuro-ophthalmological symptoms in Lyme disease remain rare but can occur. We report a case of isolated oculomotor nerve palsy due to Lyme disease with total resolution after antibiotic therapy.

Case Report: An 8 years-old boy was brought to emergencies for headaches, a left ptosis and a left exotropia. History revealed a tick bite about one year earlier during a hike in a forest followed by an erythema, non-migrant. A palsy of the third cranial nerve was confirmed by a Coordimètre Hess-Weiss test. Inflammation on the left third nerve was recorded on the cerebral MRI. Examination of the Central System Fluid (CSF) showed a lymphocytar pleocytosis ( 400 cells with $99 \%$ of lymphocytes $/ \mathrm{mm}^{3}$ ). Lyme western blot on CSF was positive. Oral doxycycline was introduced for three weeks. The follow-up was marked by total recovery of the ptosis and exotropia.

Conclusion: LNB can be responsible of acute ocular motor palsy, this infectious cause should be considered in children with acute ptosis for a prompt antibiotic treatment.
\end{abstract}

Keywords: Neuroborreliosis; Exotropia; Lyme

\section{Introduction}

Neuro-ophthalmological symptoms in Lyme disease remain rare but can occur, such as anterior uveitis, pan uveitis, acute ocular flutter, Adie's syndrome, and peripheral facial palsy [1-3]. According to the European Guidelines in Lyme neuroborreliosis, two of the three following criteria must be fulfilled to diagnose LNB: neurological symptoms, cerebrospinal fluid pleocytosis, intrathecal specific antibody synthesis [4]. We report a case of isolated oculomotor nerve palsy due to Lyme disease with total resolution after antibiotic therapy.

\section{Case Report}

\section{Clinical Features}

An 8 years-old boy was brought to emergencies for headaches, a left ptosis and a left exotropia (Figure 1). All clinical symptoms had occurred on awakening the same day after a strong fatigue the day before. No trauma was reported. His clinical history was marked by an Attention Deficit Hyperactivity Disorder (ADHD), diagnosed two years earlier and treated by Methylphenidate $20 \mathrm{mg}$ one time a day. Methylphenidate is a central nervous system stimulant, which might be associated with higher risk of strokes, though no further evidence of this association has been determined. History was eventful and revealed a tick bite about one year earlier during a hike in a forest followed by an erythema, non-migrant.

The child was living in a suburban area near a forest and his mother was working in a stable. Ophthalmic examination recorded a limitation in the field of the left medial rectus and an upper eyelid raising muscle impotence. There was no pupillary dysfunction. We noticed a conserved visual acuity (20/20 on both eyes); slit lamp bio microscopy, optic disc and retinal examination by indirect ophthalmoscopy disclosed no abnormality such as virtutis or posterior uveitis. Macular and papillary OCT B-scan (Heidelberg 
Spectral is) was normal on both eyes. A palsy of the third cranial nerve was confirmed by a Coordimètre Hess-Weiss test (Figure
2). Neurological examination, performed by a pediatrician, was normal.

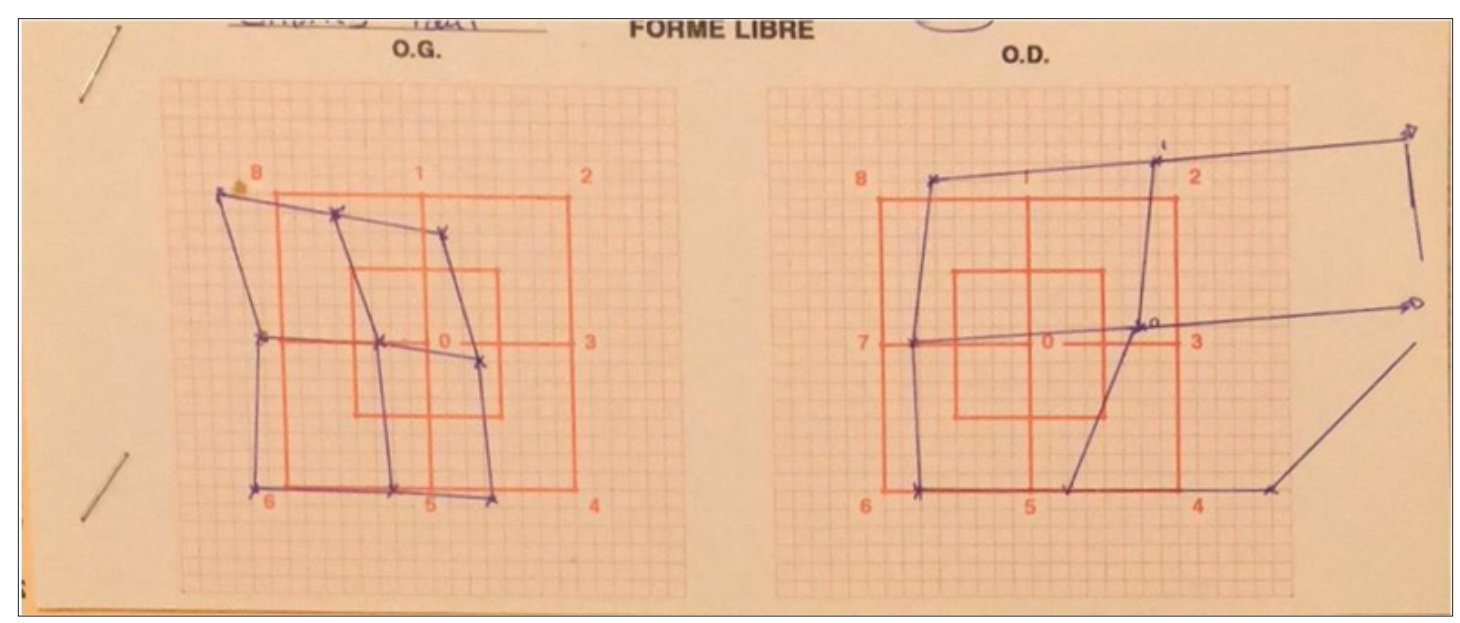

Figure 1: Initial Hess--Weiss Test.

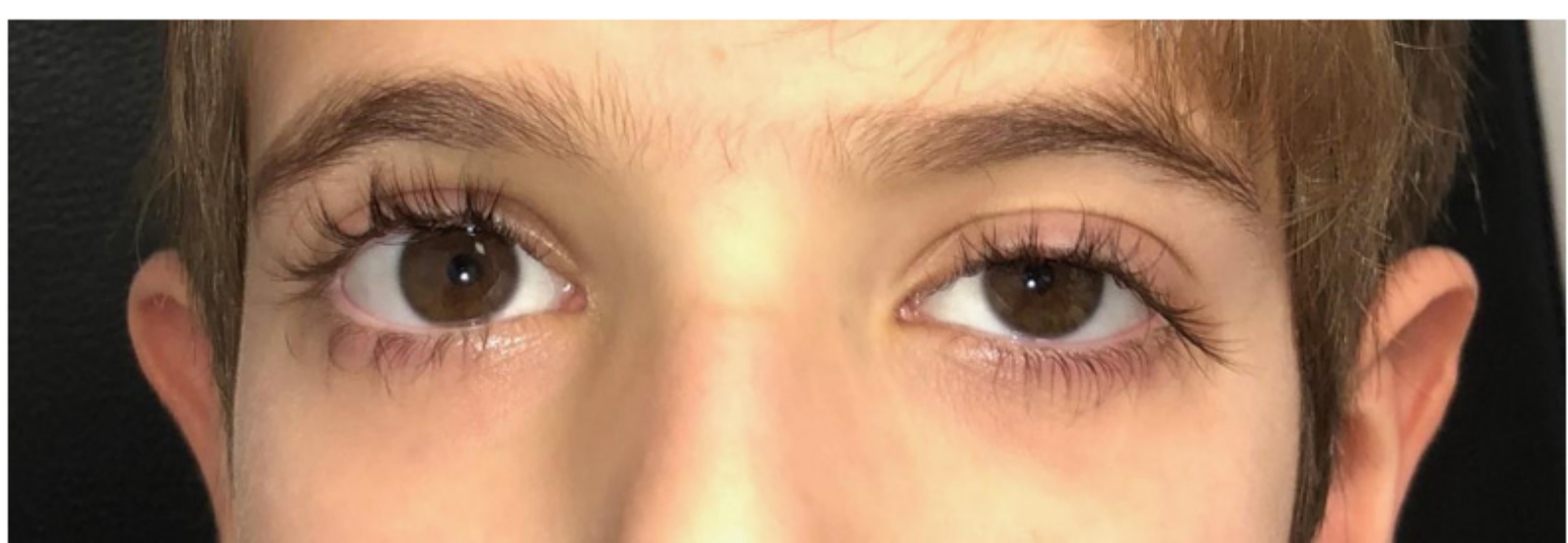

Figure 2: Initial clinical picture.

\section{Diagnostic findings}

An emergency ocular and cerebral MRI scan was performed. Inflammation on the left third nerve was recorded on the MRI (Figure 3). Complete blood count was normal; Lyme serology was negative IgG 0,01 (ELFA) and IgM 0,02 (ELFA) such as other serological results (HSV 1 and 2, VZV, Enterovirus). A lumbar puncture was performed. Examination of the Central System Fluid (CSF) showed a lymphocytar pleocytosis (400 cells with $99 \%$ of lymphocytes/mm3). Glucose and lactate were normal. CSF antiBorrelia burgdorferi IgG were negative (0,01 ELFA) and Lyme PCR was negative. Also Lyme western blot on CSF was positive.
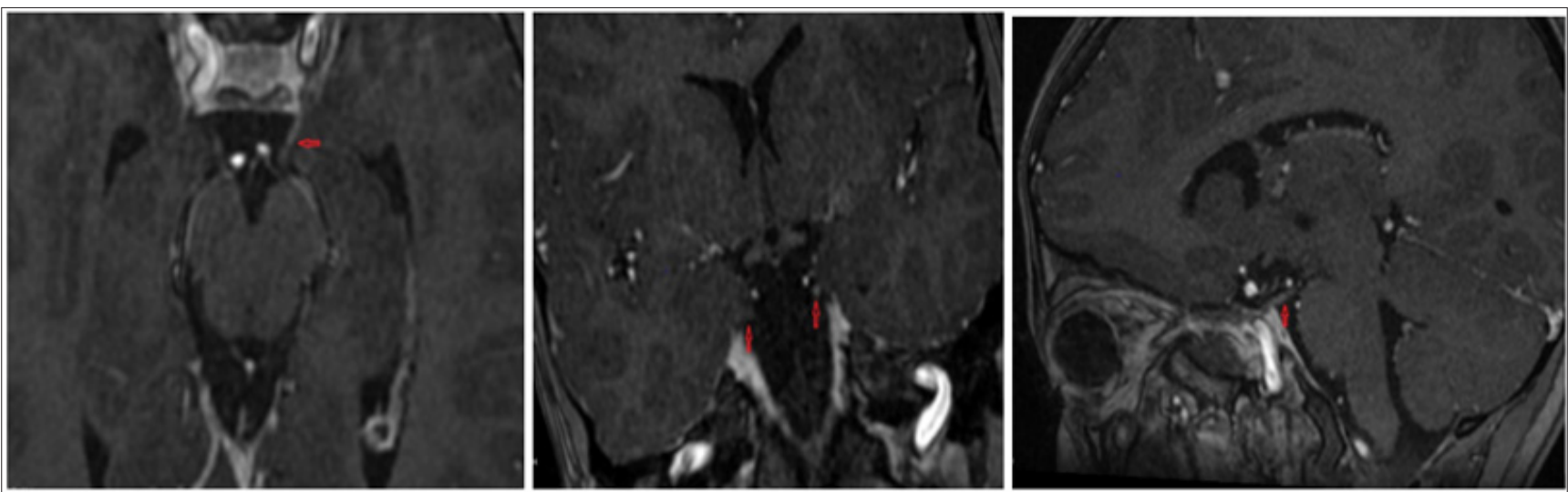

Figure 3: Initial MRI (axial, coronal, sagittal). 


\section{Therapy and Clinical Course}

The decision was made, in association with the pediatric department, to begin an oral treatment according to the patient's weight with doxycycline $100 \mathrm{mg}$ twice a day for 3 weeks. Methylphenidate was stopped and reintroduced one month later due to important behavior disorders. The ocular movement improved within a week of treatment and the Hess-Weiss test was normalized two weeks later (Figure 4). Ptosis was the only remaining symptom but receding (Figure 5). The follow-up was marked by a return to normal two months after the end of the treatment with a normal elevator muscle function.

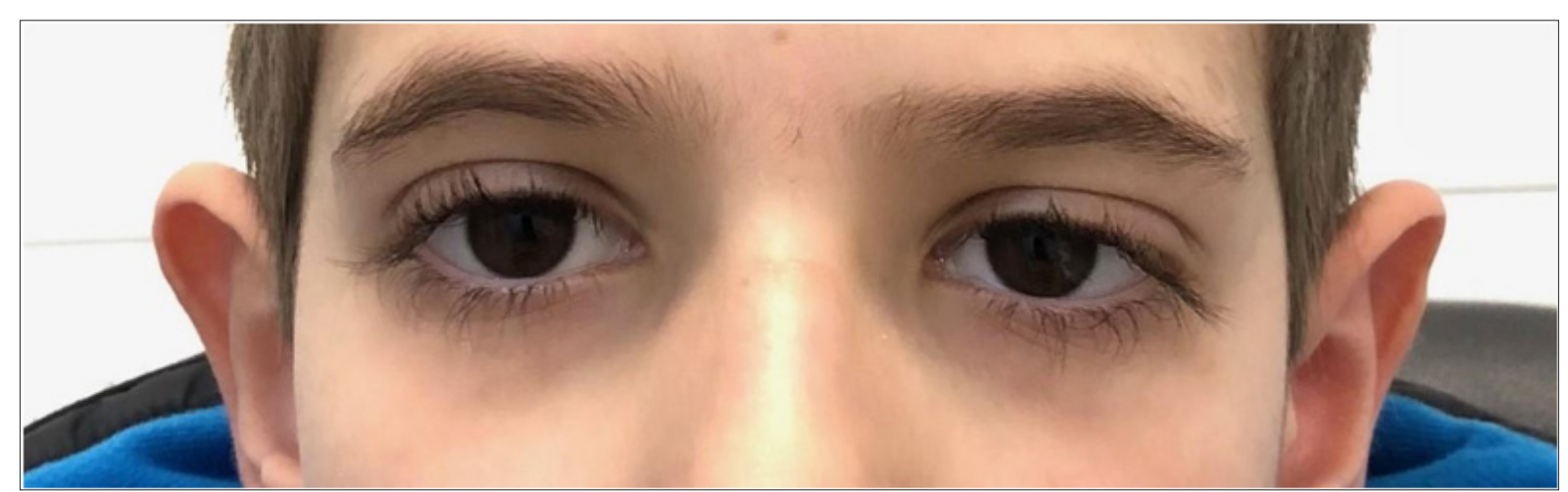

Figure 4: Follow--up clinical picture, 1 month.

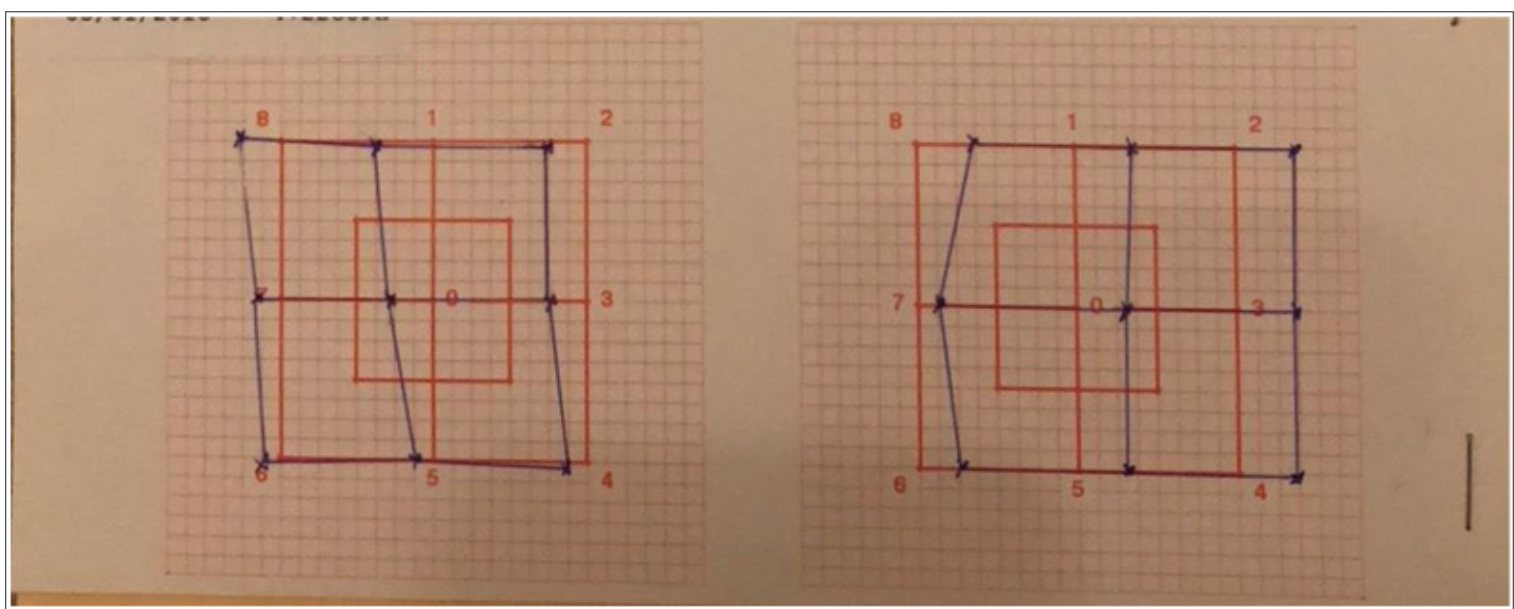

Figure 5: Follow--up Hess--Weiss Test, 2 months.

\section{Discussion}

In our case, no other cranial nerve was involved. Palsy was predominating on the upper eyelid elevator muscle and medial oculomotor muscle. Thus exotropia and diplopia were hidden. Indeed, third nerve gives innervation of the elevator muscle through its upper branch while its inferior branch innervates medial rectus. Lyme neuroborreliosis is listed as a differential diagnosis of acquired ocular motor disturbance but remains rare [5]. CSF pleocytosis and the clinical history of tick bite reported by the mother guided the diagnosis to Lyme neuroborreliosis. CSF pleocytosis allowed making the difference between LNB and orbital inflammation due to common Lyme disease. Orbital inflammation can also provide ptosis via mechanic conflict, but no abnormal findings are reported in CSF. Lumbar puncture is the gold standard for the diagnosis of LNB.

Though Lyme serologies were negative, it was not renewed during the follow up, and antibodies may be detected earlier in CSF than in serum especially in children [6]. The diagnosis of LNB must be based on a combination of various techniques; no correlation between levels of specific Borrelia Burgdorferi antibodies detected with recombinant antigen ELISA and the number of protein fractions developed with these antibodies by immunoblot has been showed [7]. Cerebral MRI is the imaging method of choice, but the raising of the nerve root is not always seen [8]. In our case, we excluded the diagnoses of stroke thanks to the early brain MRI. According to Schelleman and al, Methylphenidate is not statistically associated with an increased risk of stroke within adult population [9]. According to Shin and al, no increased risk was observed with methylphenidate exposure for ischemic stroke or heart failure, in children and young people (aged fewer than 17) treated for ADHD [10].

\section{Conclusion}

LNB can be responsible of acute ocular motor palsy, this infectious cause should be considered in children with acute ptosis for a prompt antibiotic treatment. Appropriate treatment allows a 
full recovery and persistent resolution. Exposure questioning must be recorded in the patient review.

\section{References}

1. Raja H, Starr MR, Bakri SJ (2016) Ocular manifestations of tick-borne diseases. Surv Ophthalmol 61(6): 726-744.

2. Sathiamoorthi S, Smith WM (2016) The eye and tick-borne disease in the United States. Curr Opin Ophthalmol 27(6): 530-537.

3. Lesser RL (1995) Ocular manifestations of Lyme disease. Am J Med 98(4A): 60S-62S.

4. Mygland A, Ljøstad U, Fingerle V, Rupprecht T, Schmutzhard E, et al. (2010) EFNS guidelines on the diagnosis and management of European Lyme neuroborreliosis. Eur J Neurol 17(1): 8-16, e1-4.

5. Lorenz B, Brodsky MC (2010) Pediatric Ophthalmology, NeuroOphthalmology, Genetics: Strabismus -- New Concepts in Pathophysiology, Diagnosis, and Treatment. In: Lorenz B, Brodsky MC (Eds.). Springer Science \& Business, Germany. Media pp. 237.

\section{ISSN: 2574-1241}

DOI: 10.26717/BJSTR.2019.13.002331

A Bennedjai. Biomed J Sci \& Tech Res

(C) (P) This work is licensed under Creative

Submission Link: https://biomedres.us/submit-manuscript.php
6. Stanek G, Fingerle V, Hunfeld KP, Jaulhac B, Kaiser R, et al. (2011) Lyme borreliosis: clinical case definitions for diagnosis and management in Europe. Clin Microbiol Infect Off Publ Eur Soc Clin Microbiol Infect Dis 17(1): 69-79.

7. Tylewska Wierzbanowska S, Chmielewski T (2002) Limitation of serological testing for Lyme borreliosis: evaluation of ELISA and western blot in comparison with PCR and culture methods. Wien Klin Wochenschr 114(13-14): 601-605.

8. Agarwal R, Sze G (2009) Neuro-lyme disease: MR imaging findings. Radiology 253(1): 167-173.

9. Schelleman H, Bilker WB, Kimmel SE, Daniel GW, Newcomb C, et al. (2012) Methylphenidate and risk of serious cardiovascular events in adults. Am J Psychiatry 169(2): 178-185.

10. Shin JY, Roughead EE, Park BJ, Pratt NL (2016) Cardiovascular safety of methylphenidate among children and young people with attention-deficit/hyperactivity disorder (ADHD): nationwide self-controlled case series study. BMJ 353: i2550.

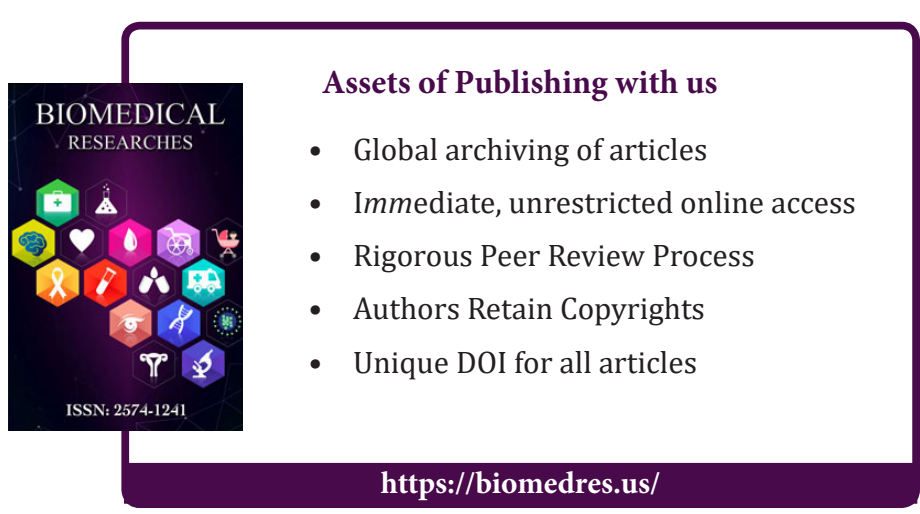

